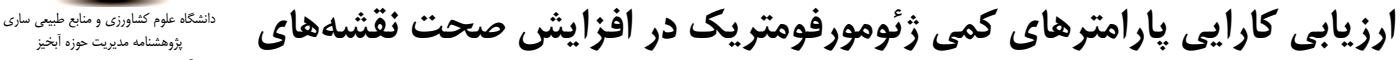

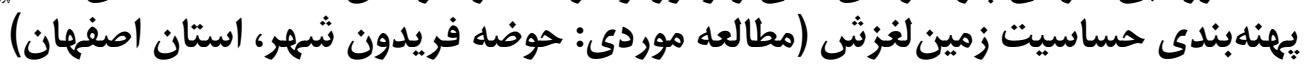

عليرضا عرب عامرى'، خليل رضايى 'مسعود سهرابى 'و كورش شيرانى

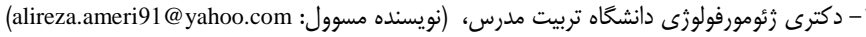

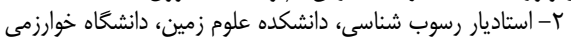

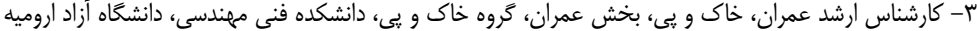

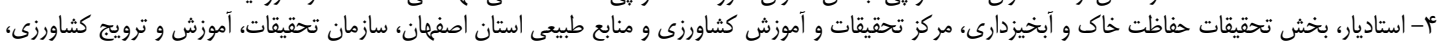
اصفهان، ايران

تاريخ دريافت:

جكيده

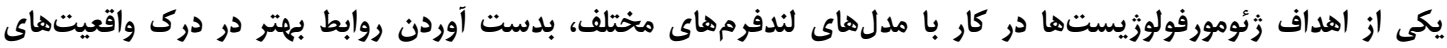

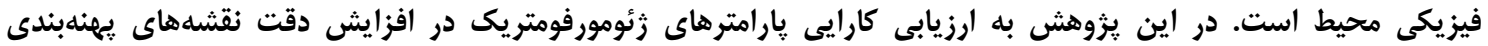

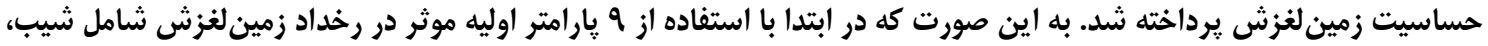

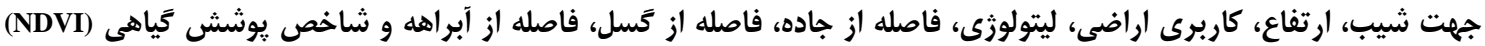

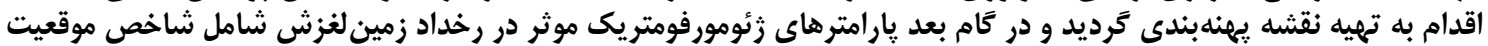

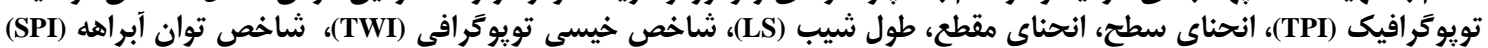

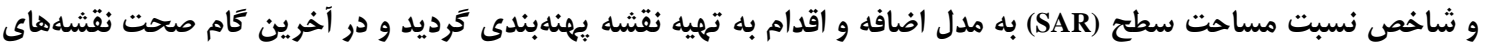

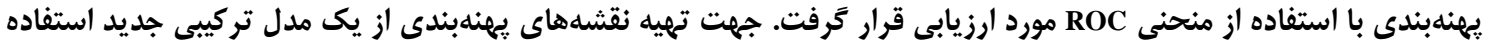

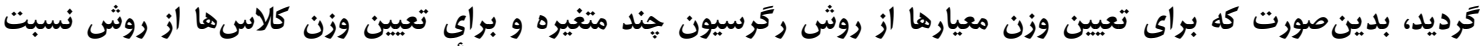

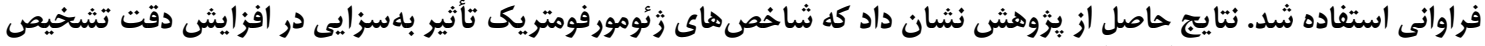

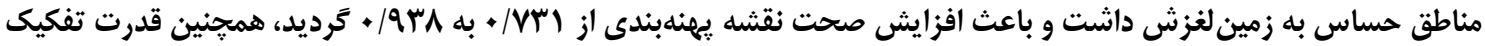

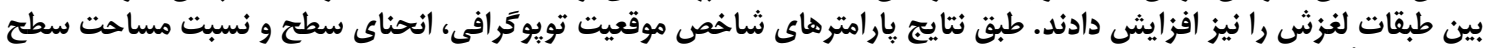

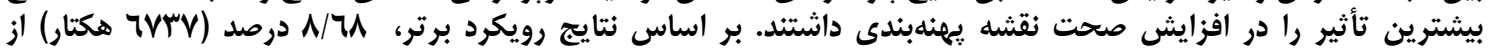

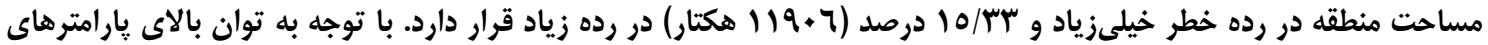

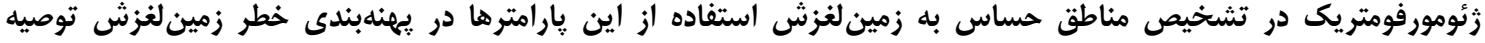

مى $\bar{~}$

وازههاى كليدى: زئومورفومترى، صحتسنجى، يهنهبندى، زمين لنزش، استان اصفهان

در اين مناطق، آن را نسبت به فجايع محيطى حساستر كرده

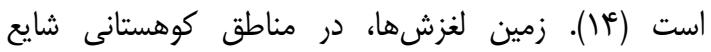

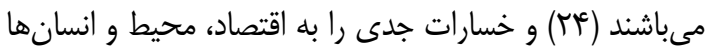

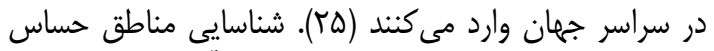

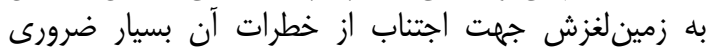

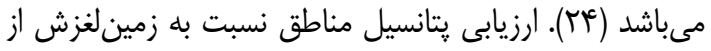

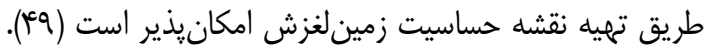

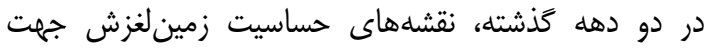

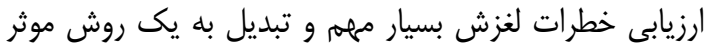

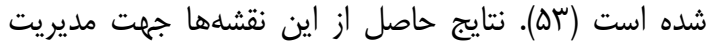

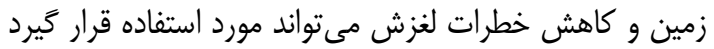

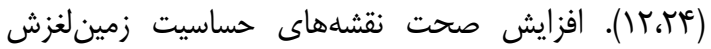

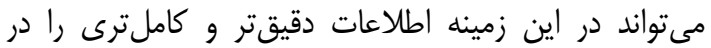

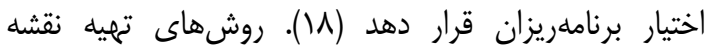

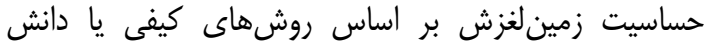

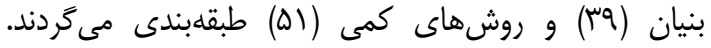

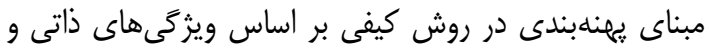

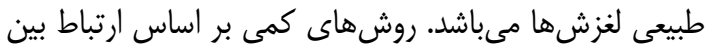

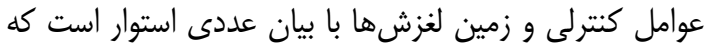

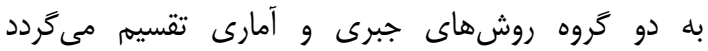

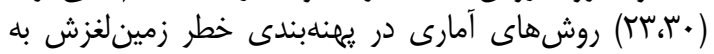

مقدمه

در زئومورفولوزى كمى ويزگى هاى فضايى و آمارى و

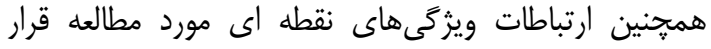

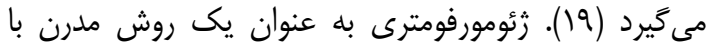

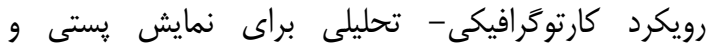

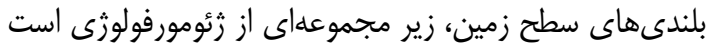

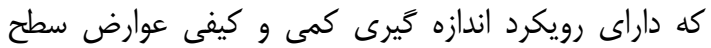

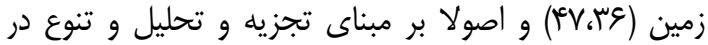

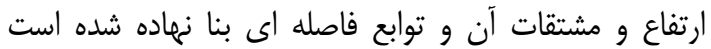

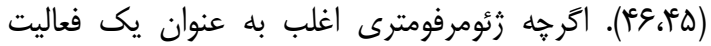

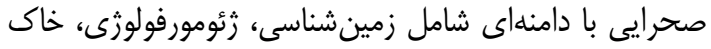

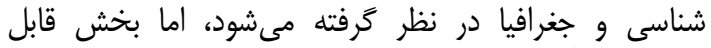
توجهى از آن شامل تكنيكهاى رافيات دايانهاى نظير سامانههاى

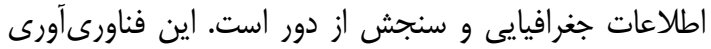

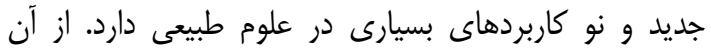

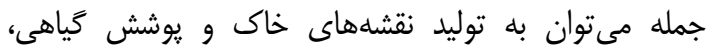

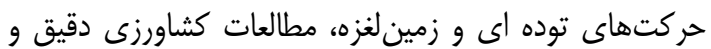

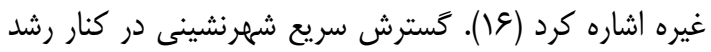

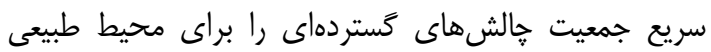

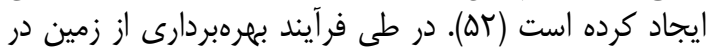

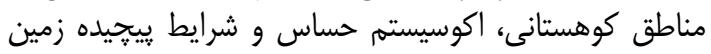


شيب، زمينشناسى، خاكشناسى، فاصله از آبراهل، فاصله از

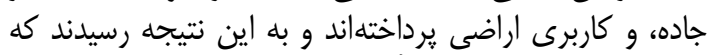

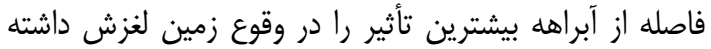

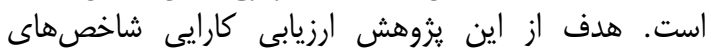

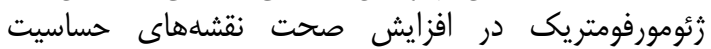
زمينلغزش مىباشد، بدينصورت دورئ كه در ابتدا با استفاده از

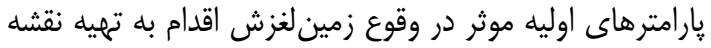

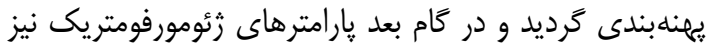

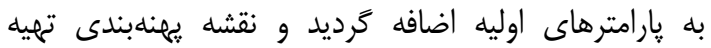

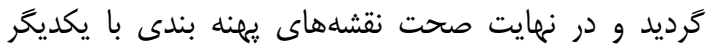

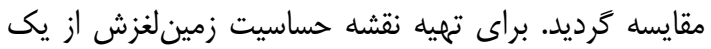

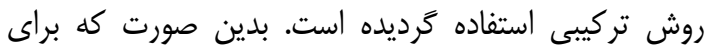

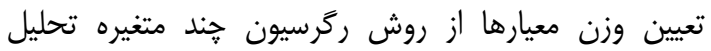

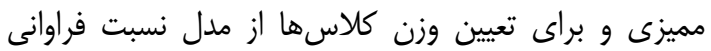

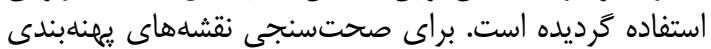

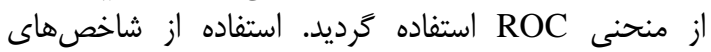

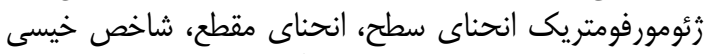

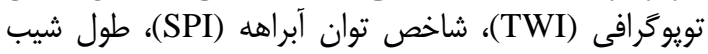

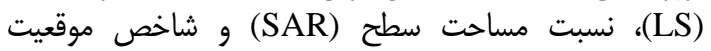

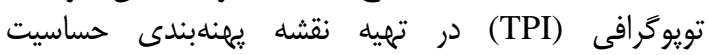
زمينلغزش و ارزيابى نقش آنها در افزايش صحت نقيه نقشههاي

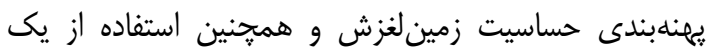

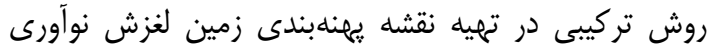
يزوهش حاضر مى باشد.

\section{مواد و روشها موقعيت منطقه مورد مطالعه}

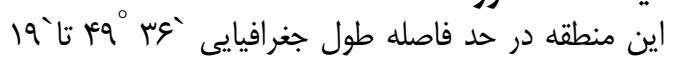

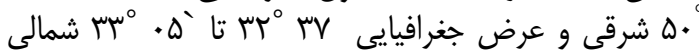

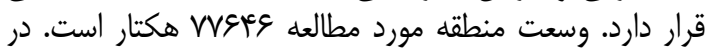

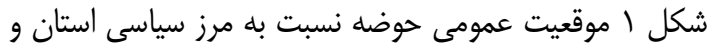

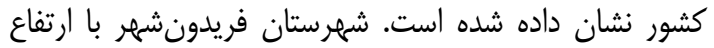

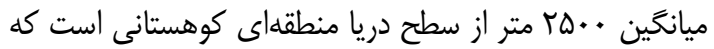

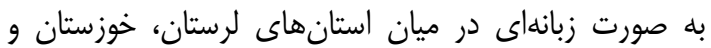

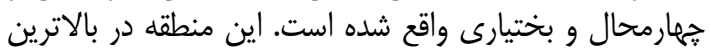

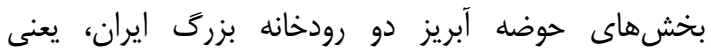

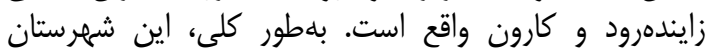

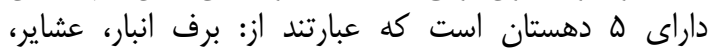

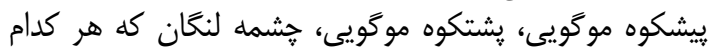

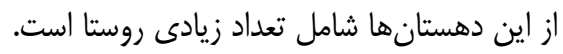

دو صورت دومتغيره و جند متغيره مورد استفاده قرار مى تىيرد.

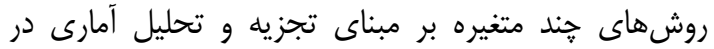

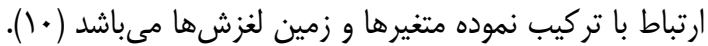

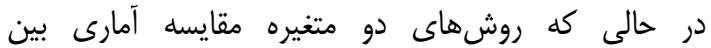

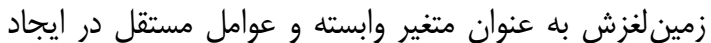

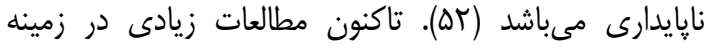

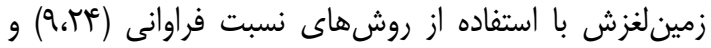

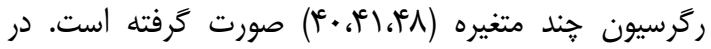

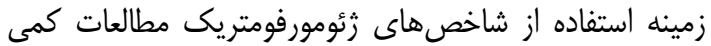

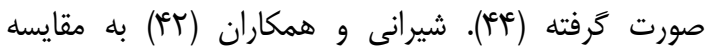

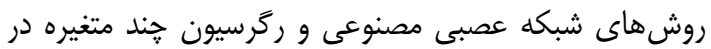

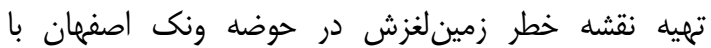

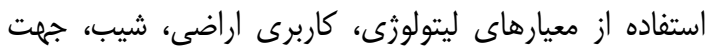

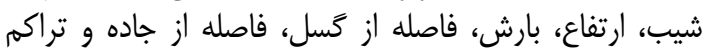

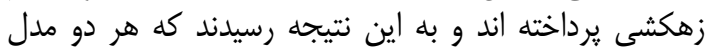

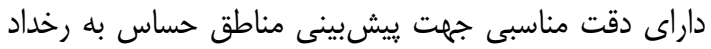

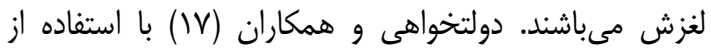

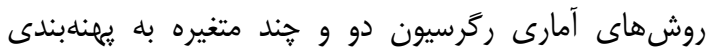

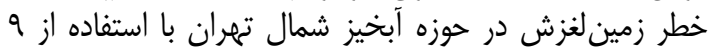

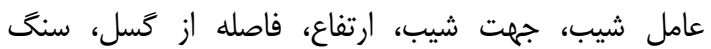

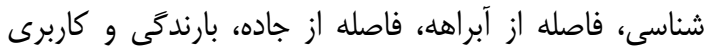

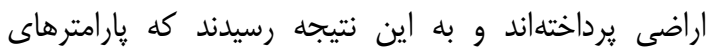

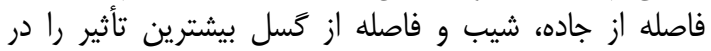

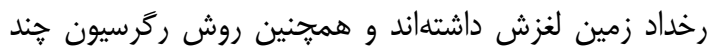

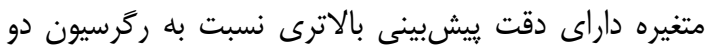

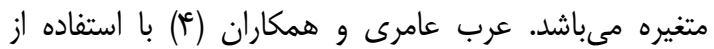

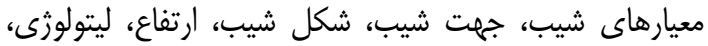

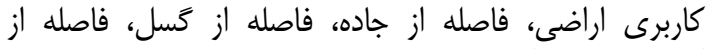

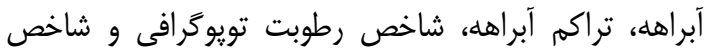

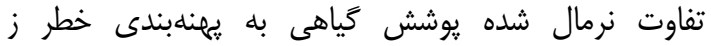

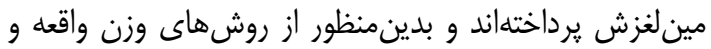

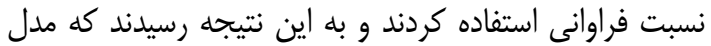

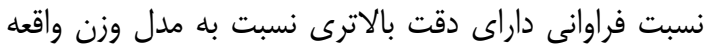

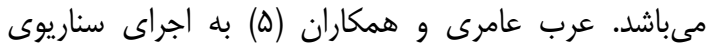
مختلف جهت تهيه نقشه حساسيت رخداد لغزش بار با استفاده از

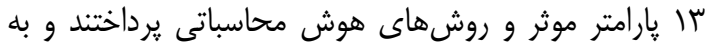

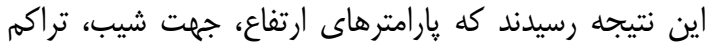

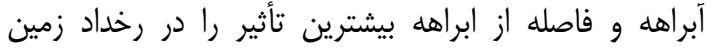

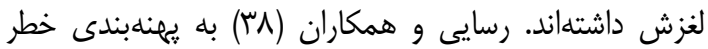

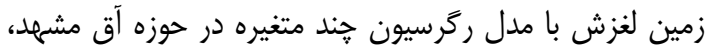

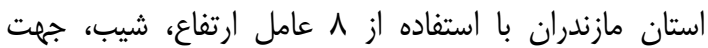




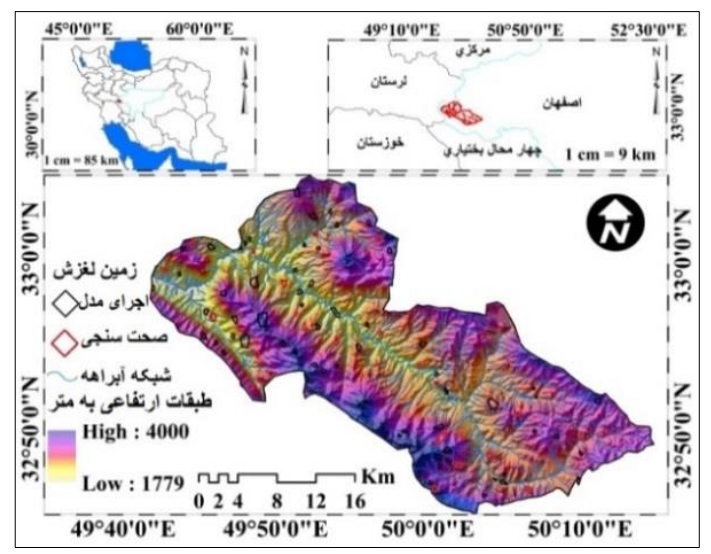

شكل 1- موقعيت منطقه مورد مطالعه

Figure 1. Area of study

ليتولوزى (شكل r. ج)، كاربرى اراضى (شكل r. ح) و شاخص

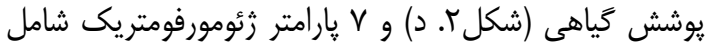

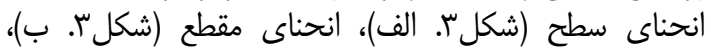

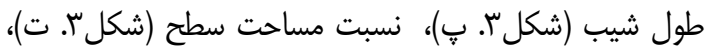

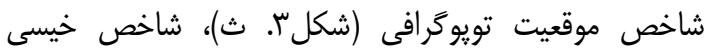

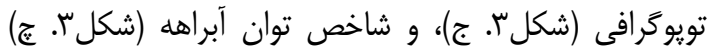
مىباشد.

\section{روش تحقيق}

نقشههاى بايه مورد استفاده در اين تحقيق عبارتند از

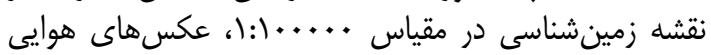

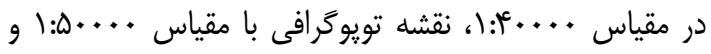

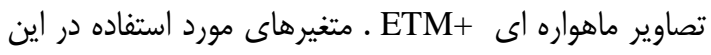

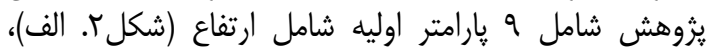

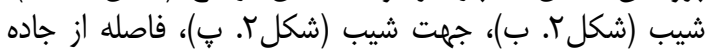

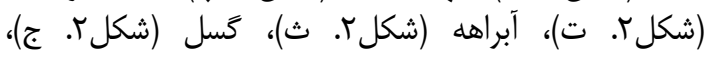

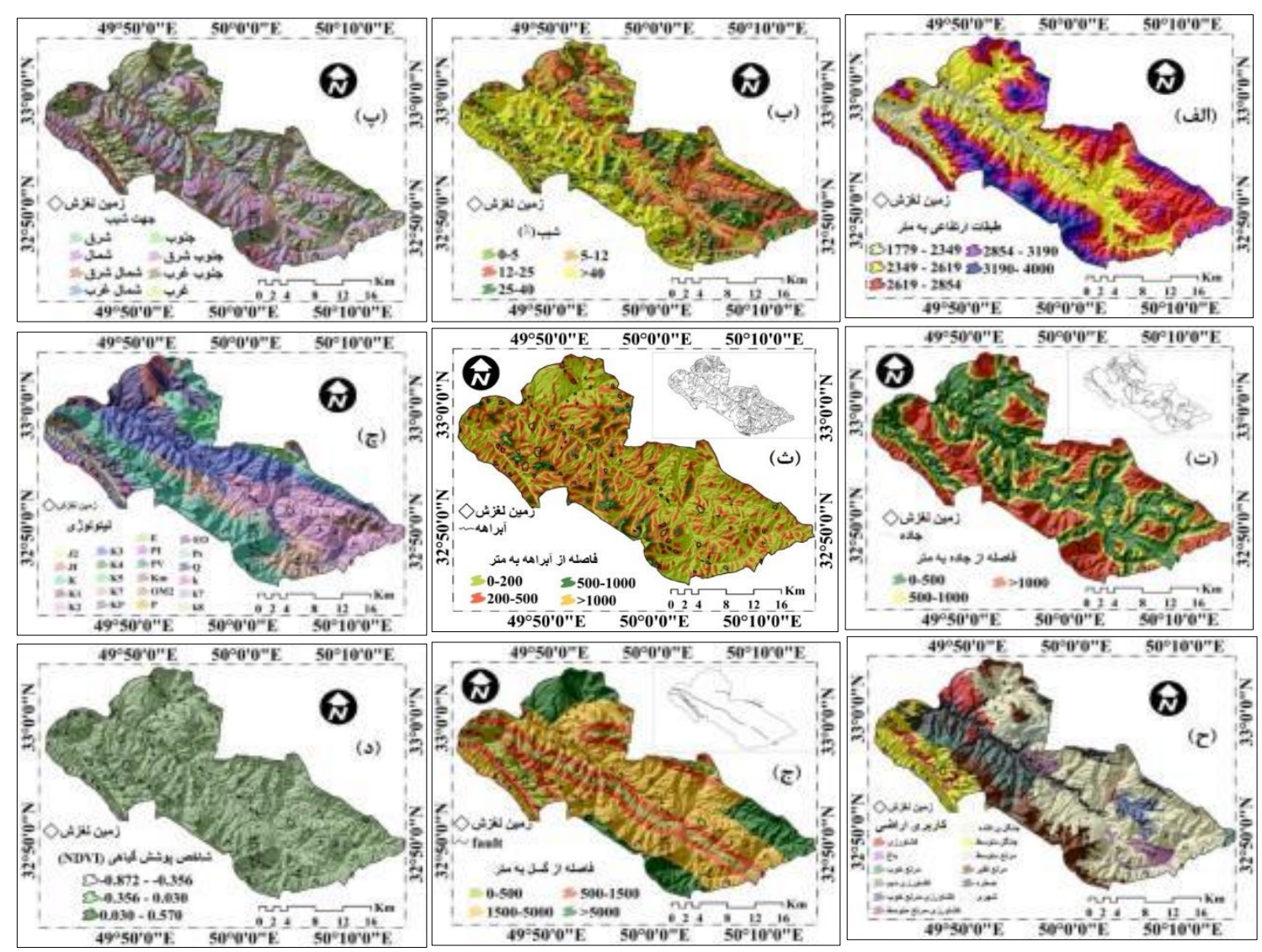

شكل r- يارامترهاى اوليه موثر در لغزش

Figure 2. Initial conditioning factors 

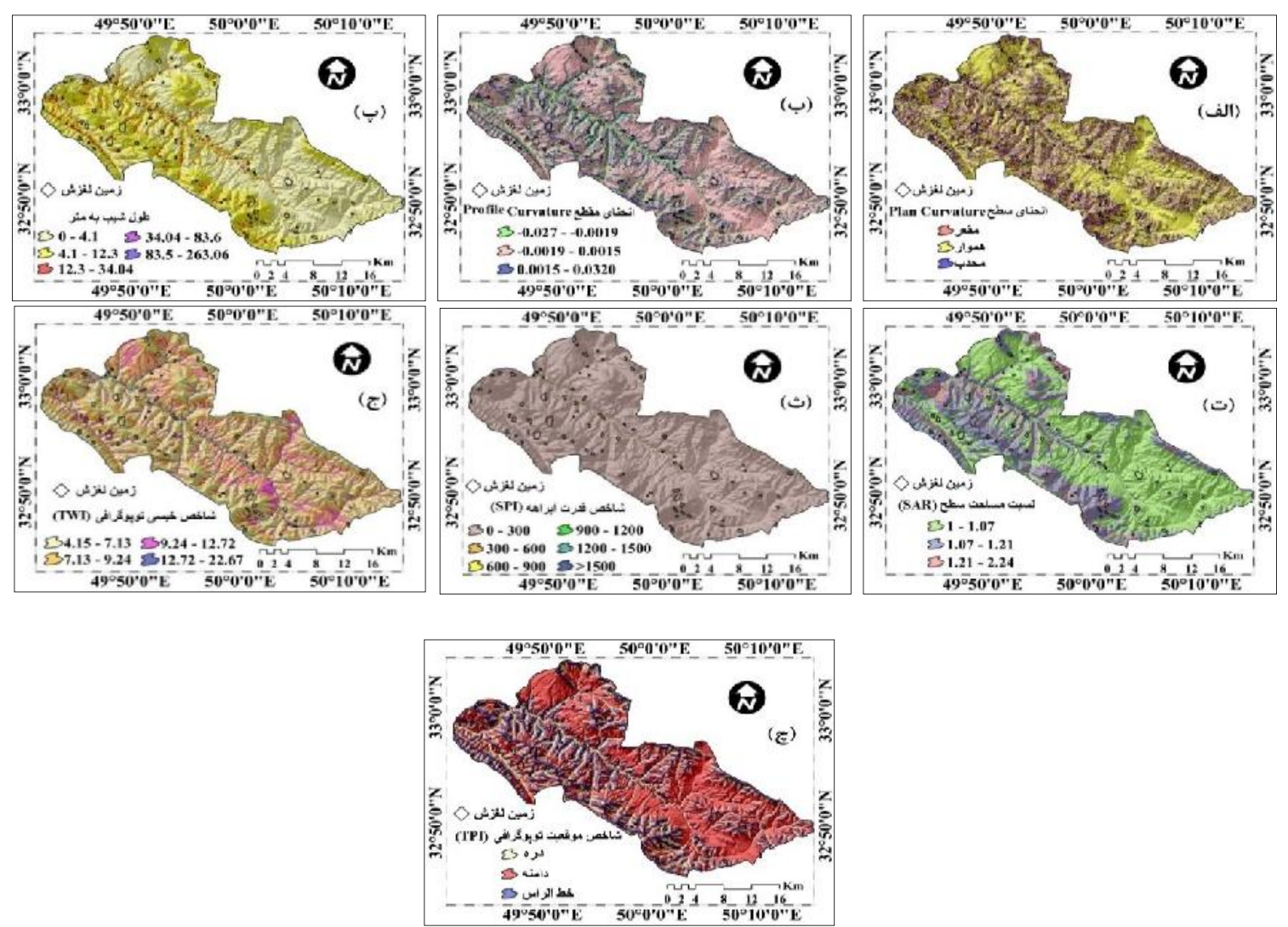

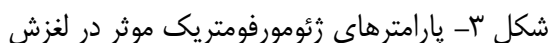

Figure 3. Geomorphometric conditioning factors

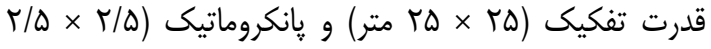

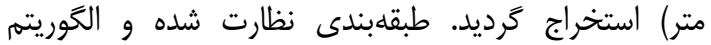

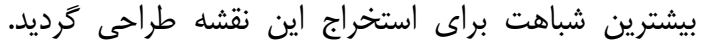

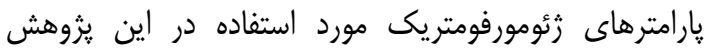

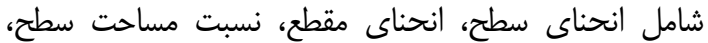

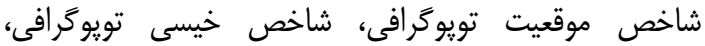

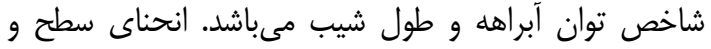

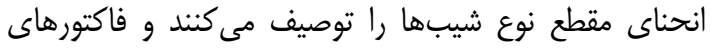

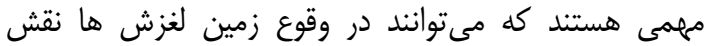

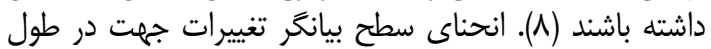

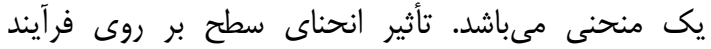
فرسايش شيب به صورت همگرايى و واگرايى آب دآن در طول

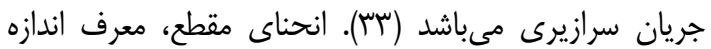

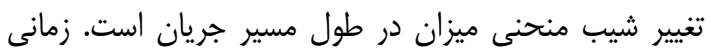
كه تقعر انحناى سطح در حال افزايش است، مقادير دادير آن منفى مانى زمانى

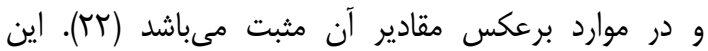

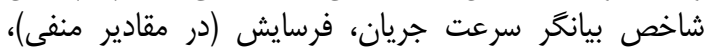

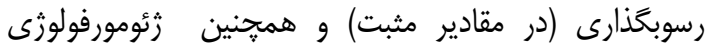

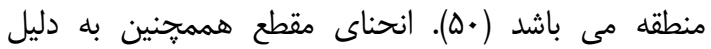

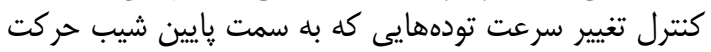

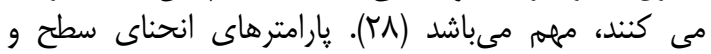

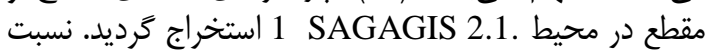

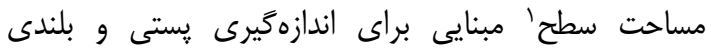

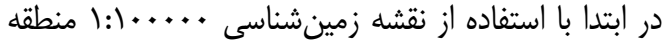

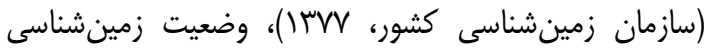

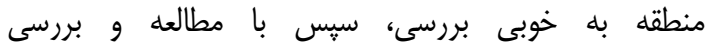

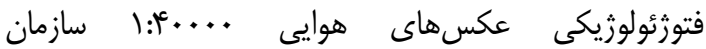

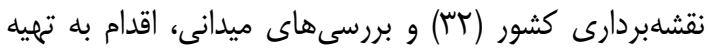

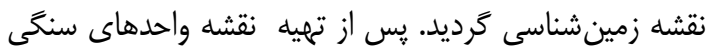

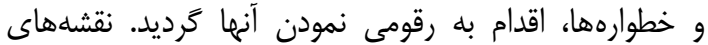

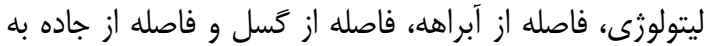

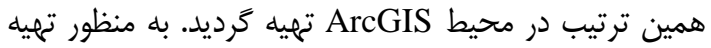

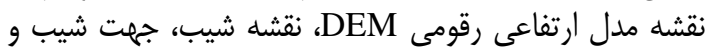

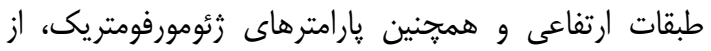

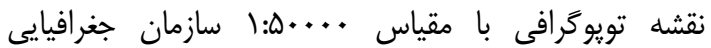

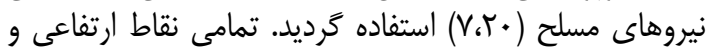

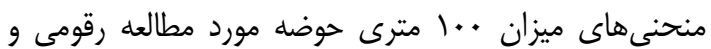

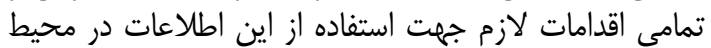

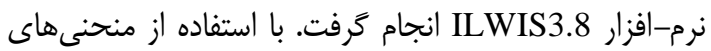

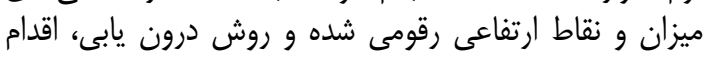

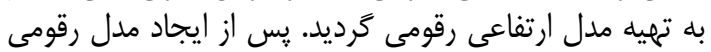

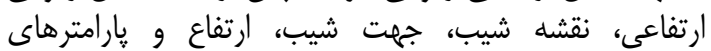

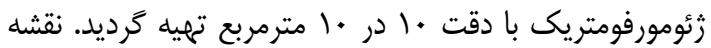

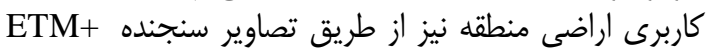

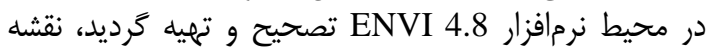

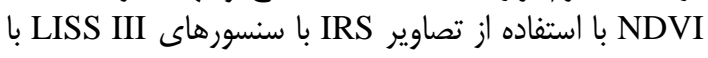




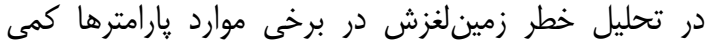

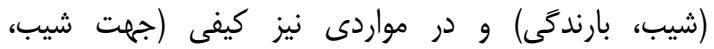

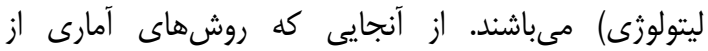

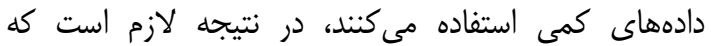

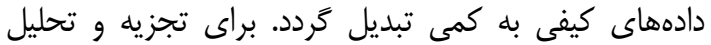

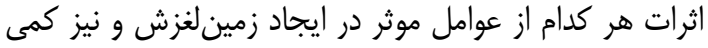

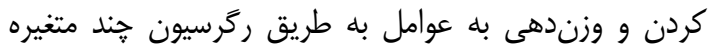

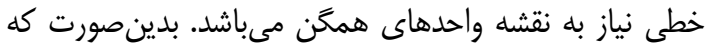

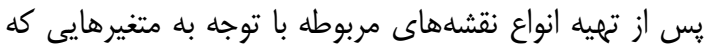

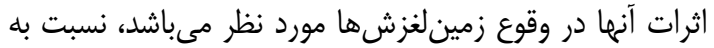

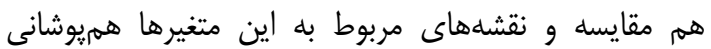

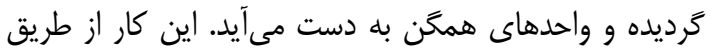

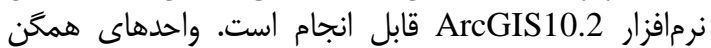

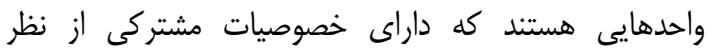

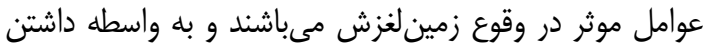

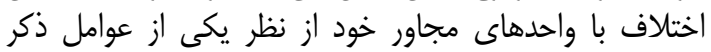

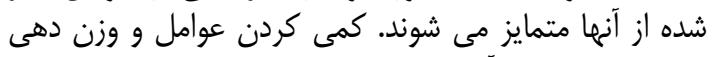

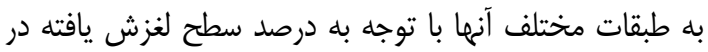

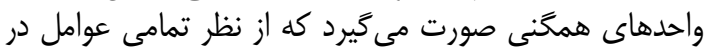

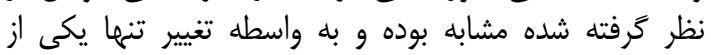
عوامل متفاوت مى باشند. به اين منظور ابته ابتدا نقشه واحدهاى

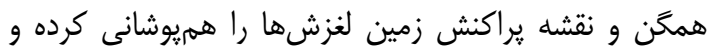

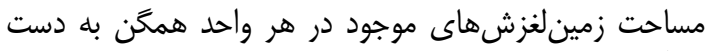

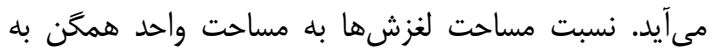
عنوان Y در نظر كرفته مى شود كه ده در واقع بيانكر درصد

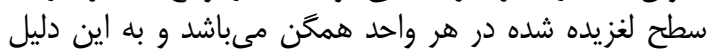

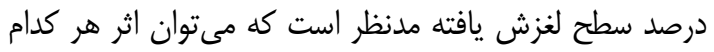

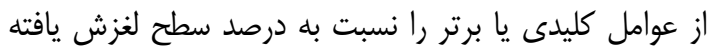

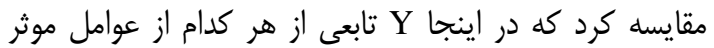

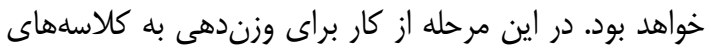

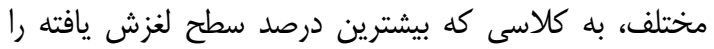

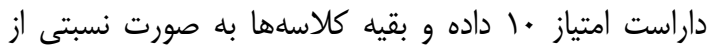

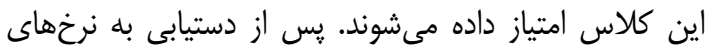

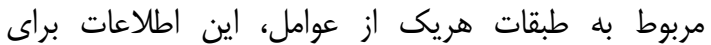


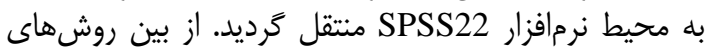

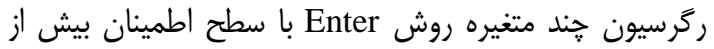

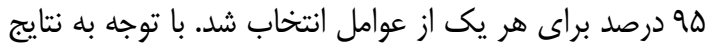

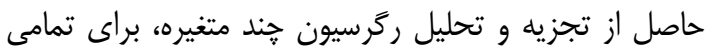

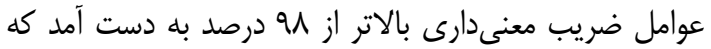

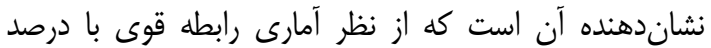
سطح لغزش يافته در واحدهاى همكَن داشته اند. روش نسبت فراوانى

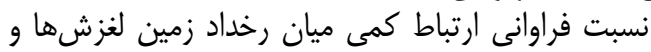

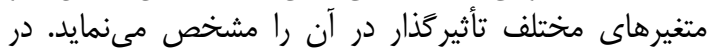

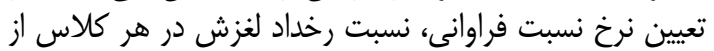

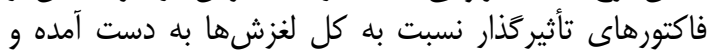

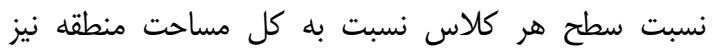

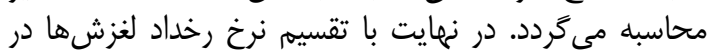

هشمانداز مىباشد. شيبهاى با يستى و بلندى بالا به دليل

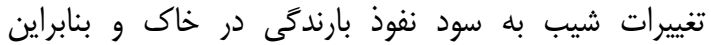

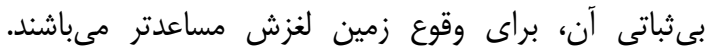
نسبت مساحت سطح هر منطقه در خشمه انداز با استفاده از

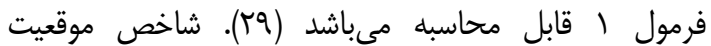

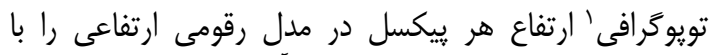

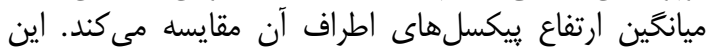

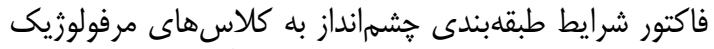

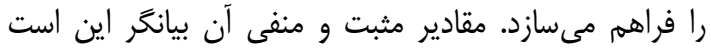

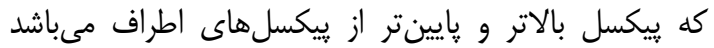

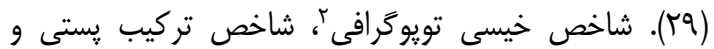

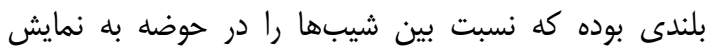

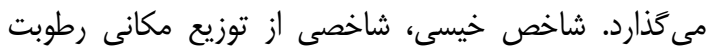

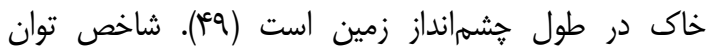

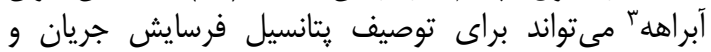

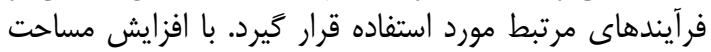

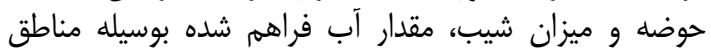

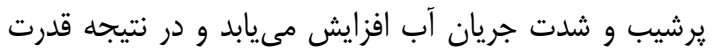

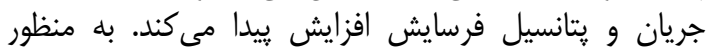
محاسبه شاخصها از معادلات زير استفاده كرديد (اسّ).

$$
\begin{aligned}
& \mathrm{SAR}=\left(\frac{\mathrm{A}}{\mathrm{A}_{\mathrm{S}}}\right) \\
& \mathrm{TWI}=\operatorname{In}\left(\mathrm{A}_{\mathrm{S}} / \tan \beta\right) \\
& \mathrm{SPI}=\mathrm{As} \times \tan \sigma \\
& \mathrm{SL}=\left(\mathrm{A}_{\mathrm{S}} / 22.13\right)^{0.6} \times(\sin \beta / 0.0896)^{1.3}
\end{aligned}
$$

كه در رابطه يك A مساحت سطح منطقه به كيلومتر مربع، و

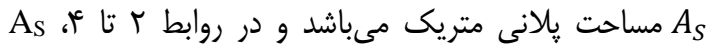

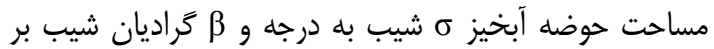
حسب درجه مىباشد. روش جند متغيره خطى منى

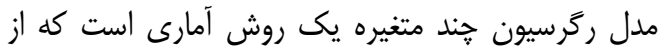

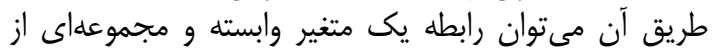

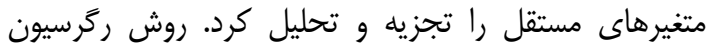

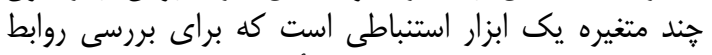

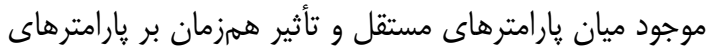

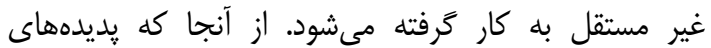

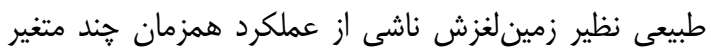

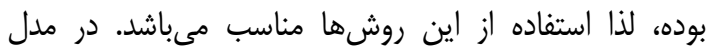

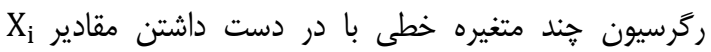

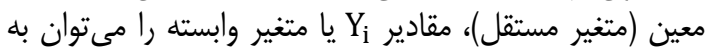

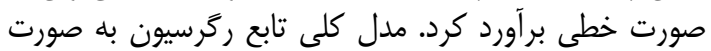

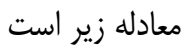

$\mathrm{Y}_{\mathrm{i}}=\mathrm{B}_{1}+\mathrm{B}_{\mathrm{r}} \mathrm{X}_{\mathrm{i}}+\cdots \cdot+\mathrm{B}_{\mathrm{j}} \mathrm{X}_{\mathrm{i}}$

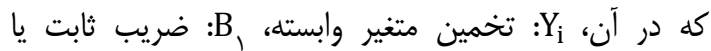

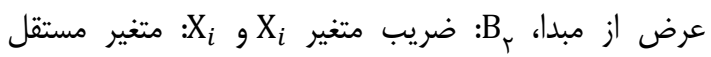

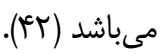




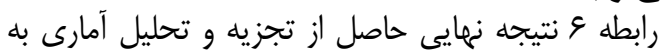

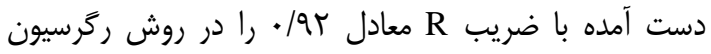

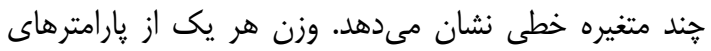

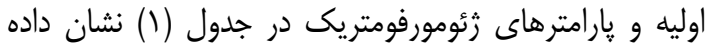

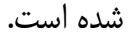
(رابطه و (ر) $Y=\cdot / \cdot 9 \mu+($ Xaltitute $X \cdot / r)+($ Xslope $X \cdot / / r \Delta)+$ $($ Xaspect $\times \cdot / / \mu \Delta)+($ Xdisfault $\times \cdot / \cdot V \Delta)+$ $($ Xdisdrinage $X-\cdot / \cdot r \mu)+($ Xdisroad $X-\cdot / \cdot \cdot / \mu)+$ $($ XNDVI $\times \cdot / \cdot F \Psi a)+($ Xlithology $\times \cdot / \Delta \cdot \mu)+$ (Xlanduse $x \cdot / \cdot \Lambda r r)+($ XSPI $x \cdot / r \cdot \Delta)+($ XLS $\times$ $. / / 9 \Lambda)+($ XTWI $X-. / . /$ M $)+($ XProfile $X-\cdot / r 9 \Delta)$ $+($ XTPI $x \cdot$ • Q - /

نتايج حاصل از تعيين ميزان اهميت معيارها در رخداد

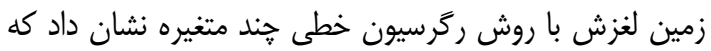

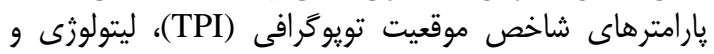

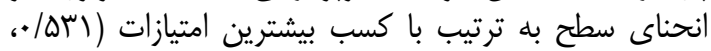

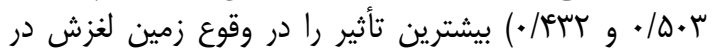

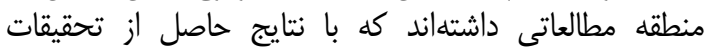

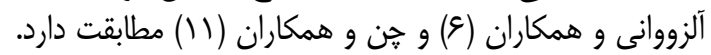

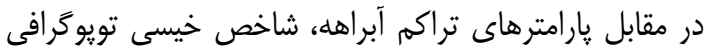

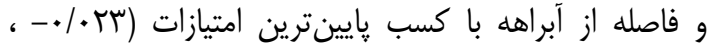

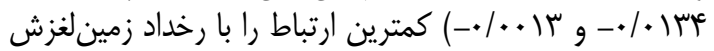

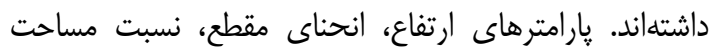

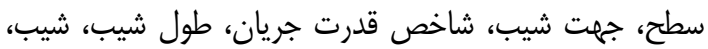

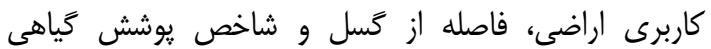

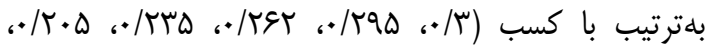

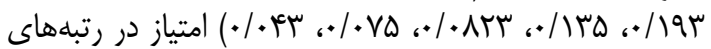

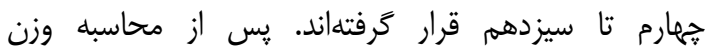

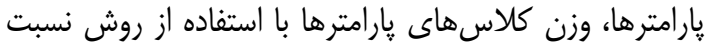

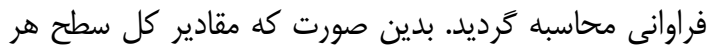

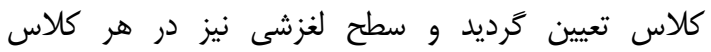

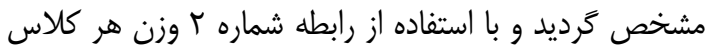

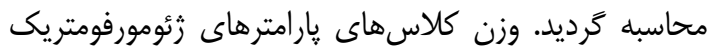

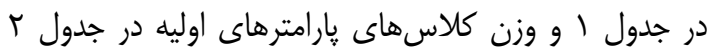

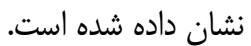

هر كلاس بر نرخ مساحت هر كلاس نسبت به كل منطقانه

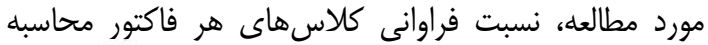

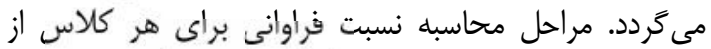
فاكتورهاى موثر در لغزش در رابطه ثُ بيان شده است:

$$
F R=\frac{(A / B)}{(C / D)}=\frac{E}{F}
$$

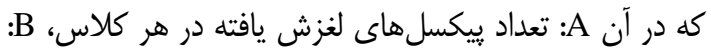

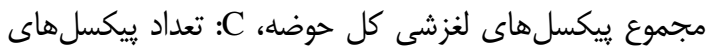

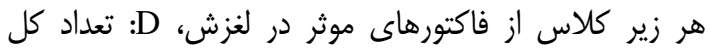

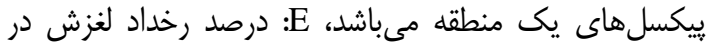

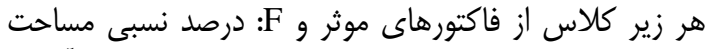

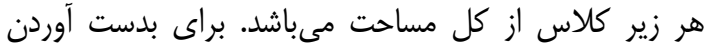

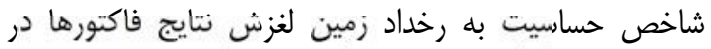
محيط GIS با يكديغر جمع مى مَردد (19).

$$
L S I=\sum(F R)_{i} \quad(i=1,2, \ldots, n)
$$

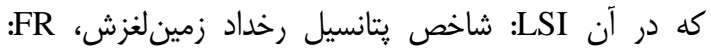

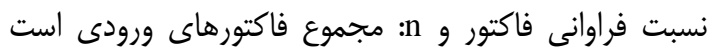

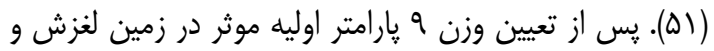

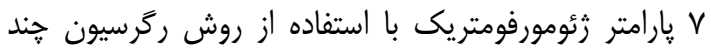

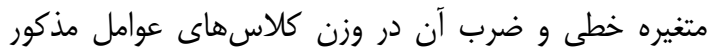

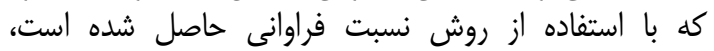

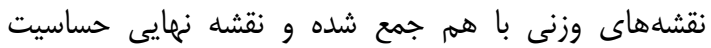

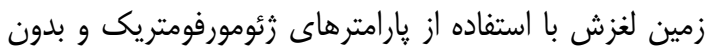

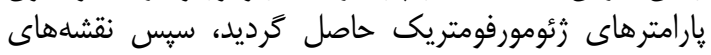

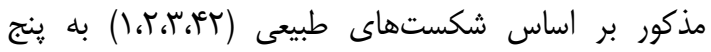
كلاس خيلىزياد، زياد، متوسط، كم و خيلى خيلى كم تقسيمبندي

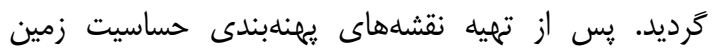

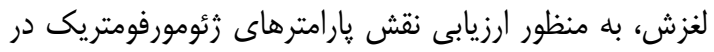

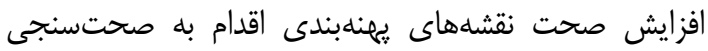

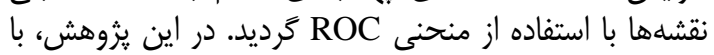

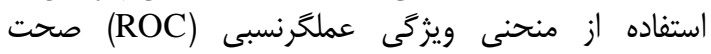

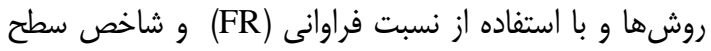

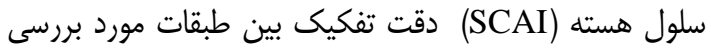

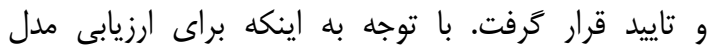

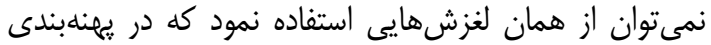

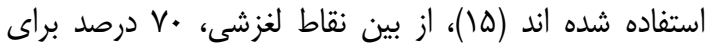
اجراى مدل و •־ درصد براى ارزيابى مدل مورد استفاده قرار

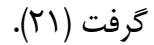


جدول ا- وزن يارامترهاى زئومورفومتريك موثر در لغزش به همراه كلاسهاى آنها Table 1. Weight of Geomorphometric conditioning factors along with their classes

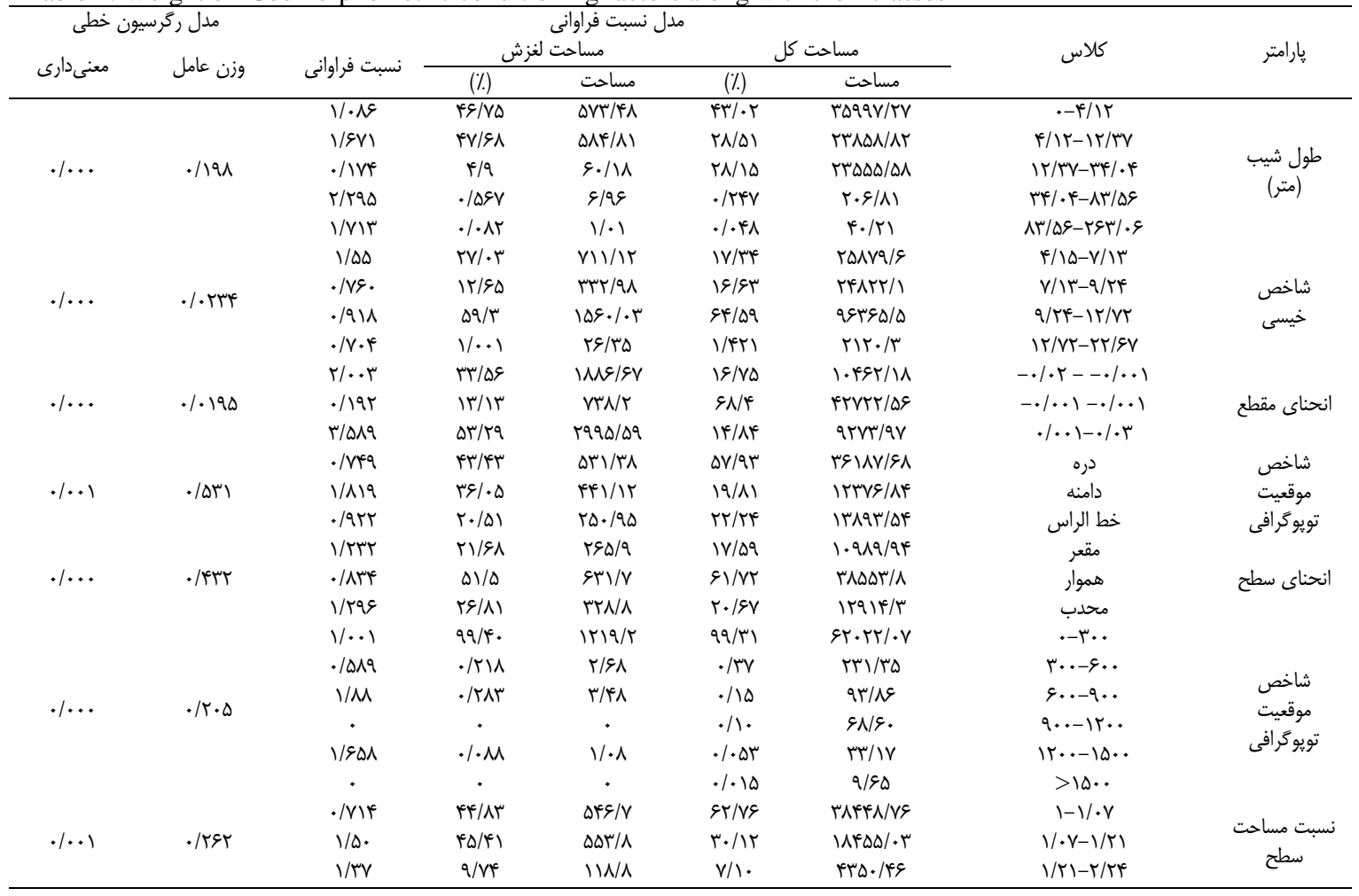

جدول r- وزن پارامترهاى اوليه موثر در لغزش به همراه كلاسهاى آنها

Table 2. weight of Initial conditioning factors along with their classes

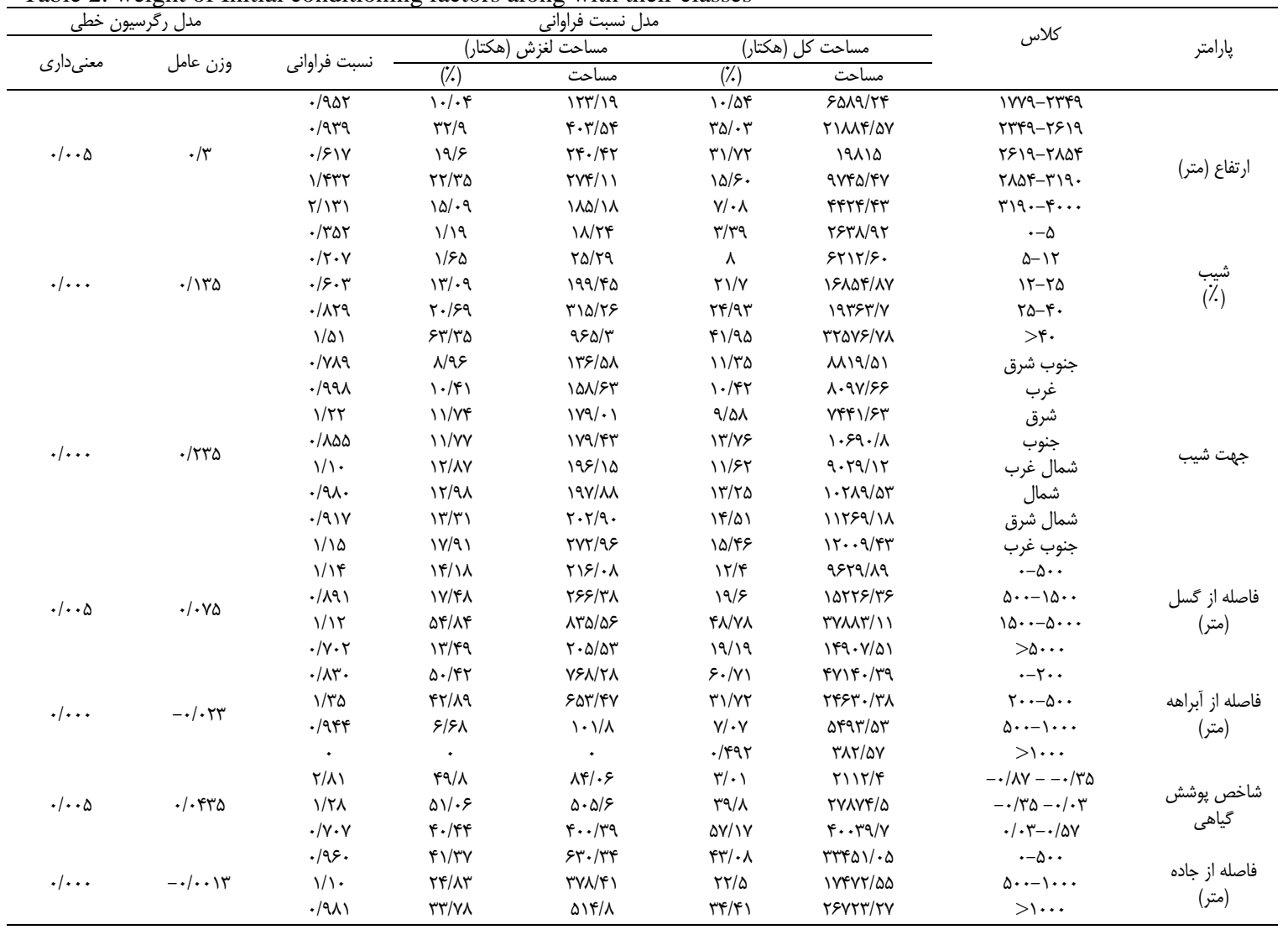


Table 2. Continue

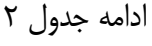

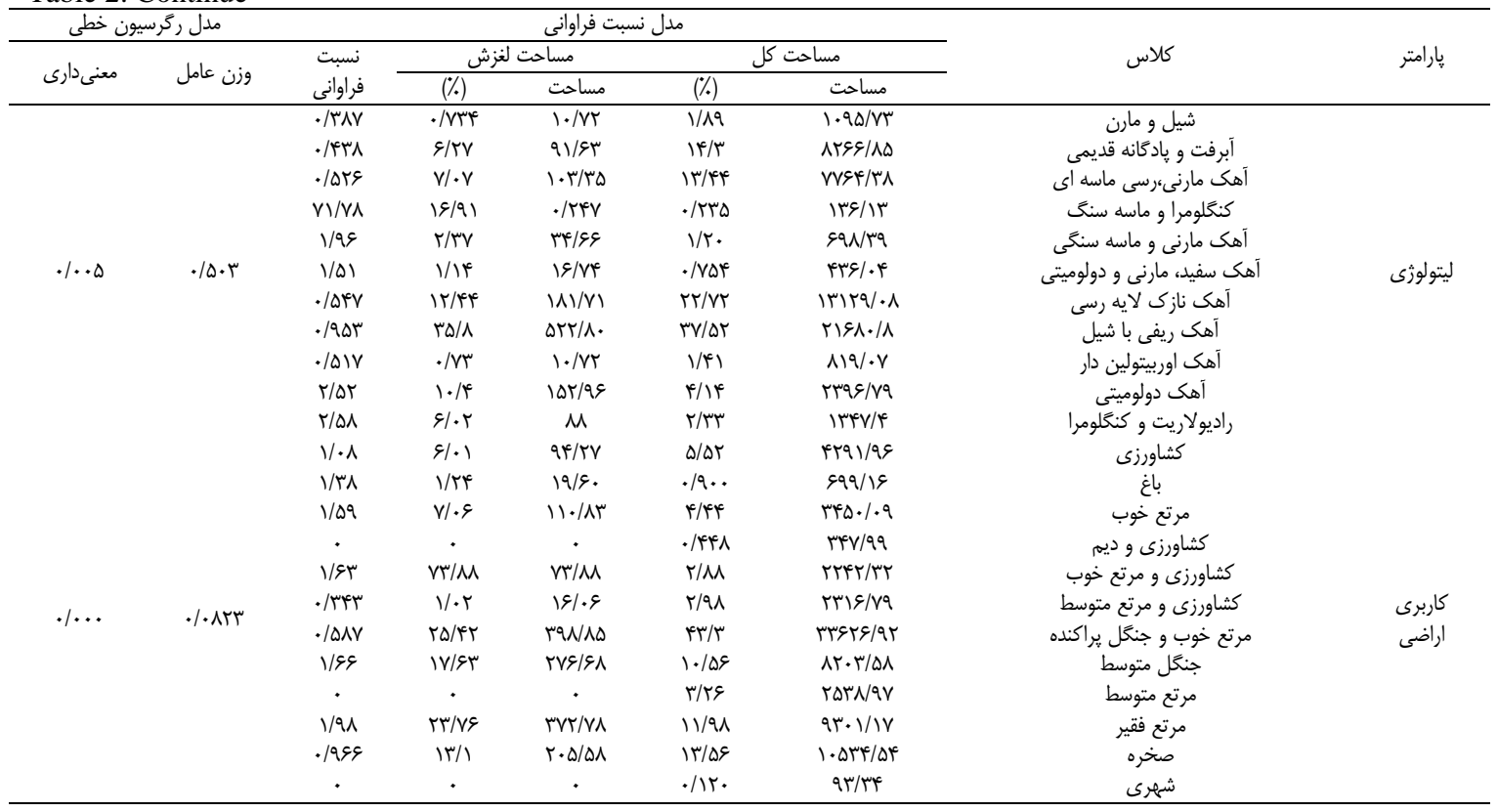

حساسيت خيلى كم، كم و متوسط قرار گرفته است. نتايج

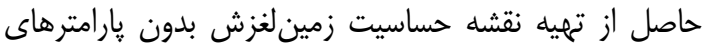

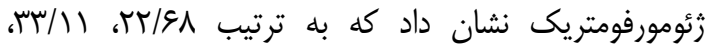

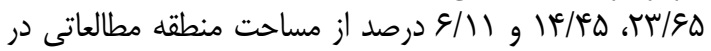

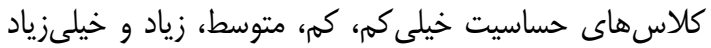
قرار كرفته است و در مقابل

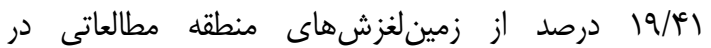

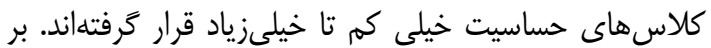

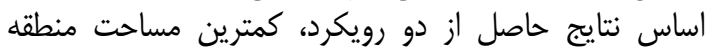

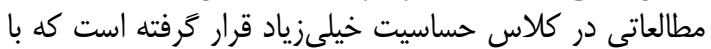

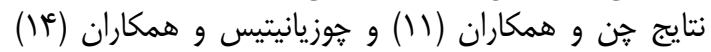
مطابقت دارد.

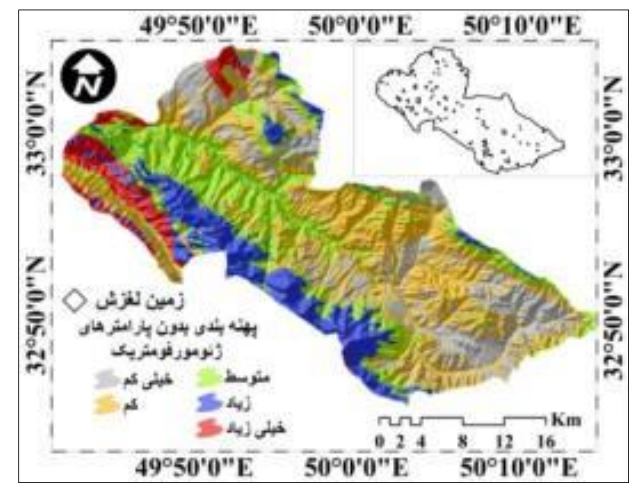

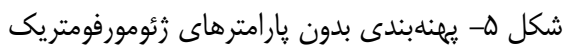
Figure 5. Zonation without Geomorphometric Factors
نتايج حاصل از يهنهبندى خطر زمينلغزش همراه باه باه

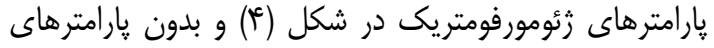

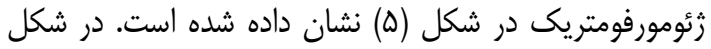

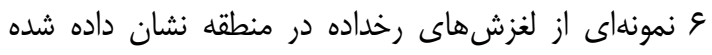

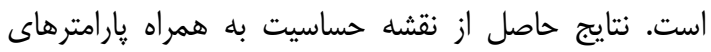

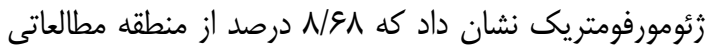

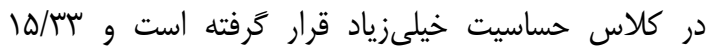

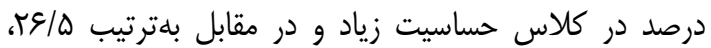

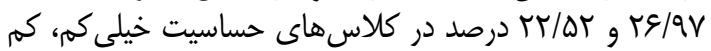

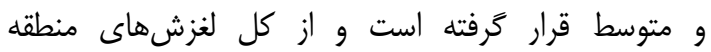

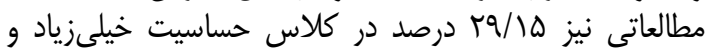

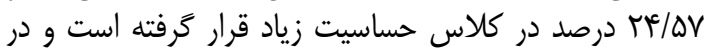
مقابل بلترتيب دq/

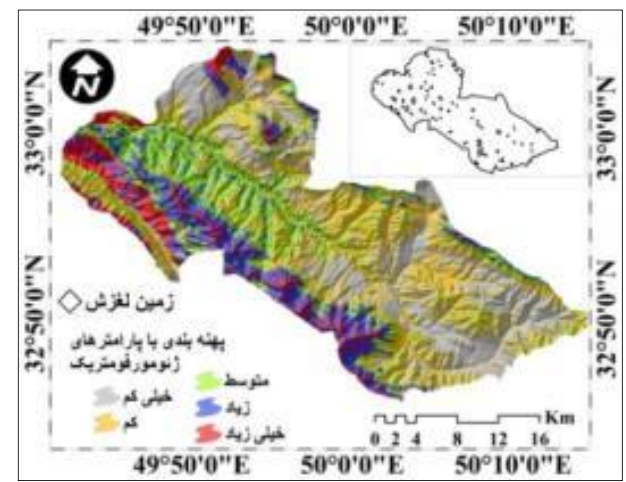

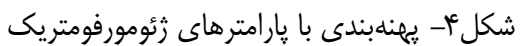

Figure 4. Zonation with Geomorphometric Factors 


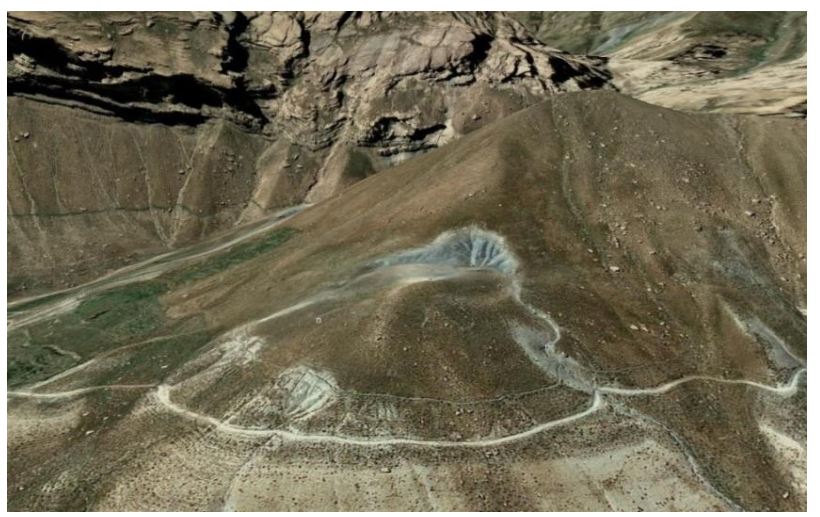

شكل \&- نمونهاى از لغزش هاى منطقه مورد مطالعه

Figure 6. A sample of landslides in the study area

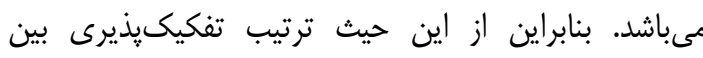

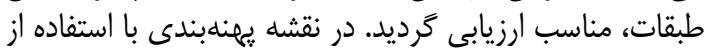

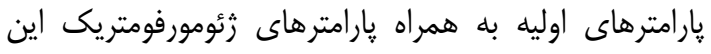

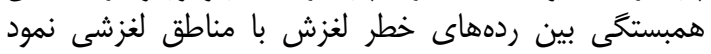

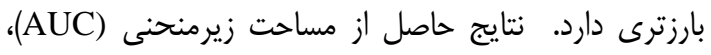

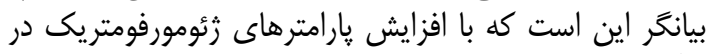

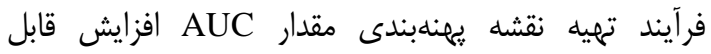

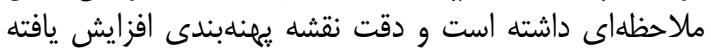

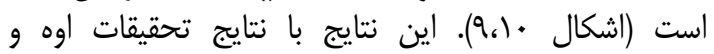

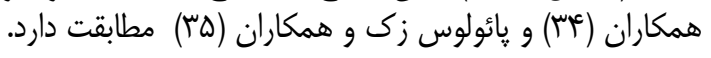

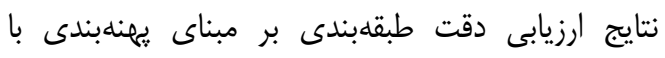

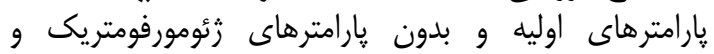

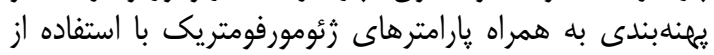

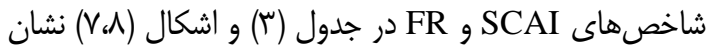

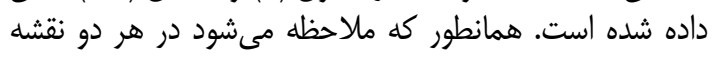

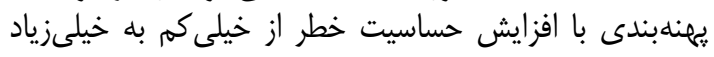

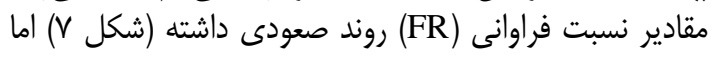

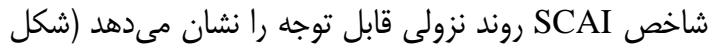

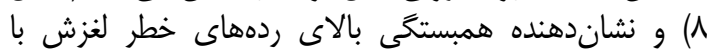
مناطق لغزشى موجود و بازديدهاى ميدانى منطقه مورد مطالعه

جدول سـ- مقادير نسبت فراوانى و شاخص سطح سلول هسته مناطق حساس زمين لغزش Table 3. Amount of Frequency Ratio and Seed Cell Area Index in landslide susceptibility areas

\begin{tabular}{|c|c|c|c|c|c|c|c|c|c|}
\hline SCAI & درصد Seed & فراوانى نسبت & مساحت رده درص & رساحت هر رهار & لغزاحت & مساحت & مساحت فاقد & ردهاى خطر & مدل \\
\hline $9 / 11$ & $r / V$ & $.1 \cdots$ & $T \& / D$ & $r \cdot \Delta V Q / \zeta$ & $1 / \wedge q$ & $V F / \Delta T$ & $r \cdot \Delta \cdot 1 / l$ & خيلى كم & \multirow{3}{*}{ هارامترهاه با } \\
\hline $1 / 99$ & $1 \% / \Delta$ & $.1 \cdot r$ & Tr/QT & IVFAN/G & $r \cdot / \Lambda r$ & TIV/TA & $|V| V \mid / e$ & متوسط & \\
\hline .190 & rm/F & . & سז/هו & 119.9 & $r F / \Delta V$ & TVE/Tr & IIUI/V & زياد & \\
\hline V/VG & $r / 9 T$ & $\cdot 1 \cdot+f^{c}$ & Tr/GA & $1 V 9.9$ & $f / \& V$ & $x / .9$ & IVDTV/Q & خيلى كم & \multirow{5}{*}{ بارامترها } \\
\hline$r / 9 \Lambda$ & $11 / 11$ & $.1 \cdot 10$ & II/Mr & $r \Delta V \cdot \varepsilon / r$ & $r \Delta / Q$ & rqp/s & rQTII/S & كم & \\
\hline l/Ar & Ir & $.1 \cdot 11$ & $r$ rT/ D & ג & $r \mid / 9$ & rrq/q & Q & متوسط & \\
\hline . $/ 0 T$ & TV/M & $.1 .4 q$ & $\mid f / F \Delta$ & $11 T r \cdot / F$ & $r N / T V$ & RTt/ & $1 \cdot V M N / 1$ & زياد & \\
\hline.$/ 1 f$ & $r Q / 1$ & .1 .94 & $9 / 11$ & FVEe/l & $19 / 41$ & r৭০/s & FETAND & خيلى زياد & \\
\hline
\end{tabular}




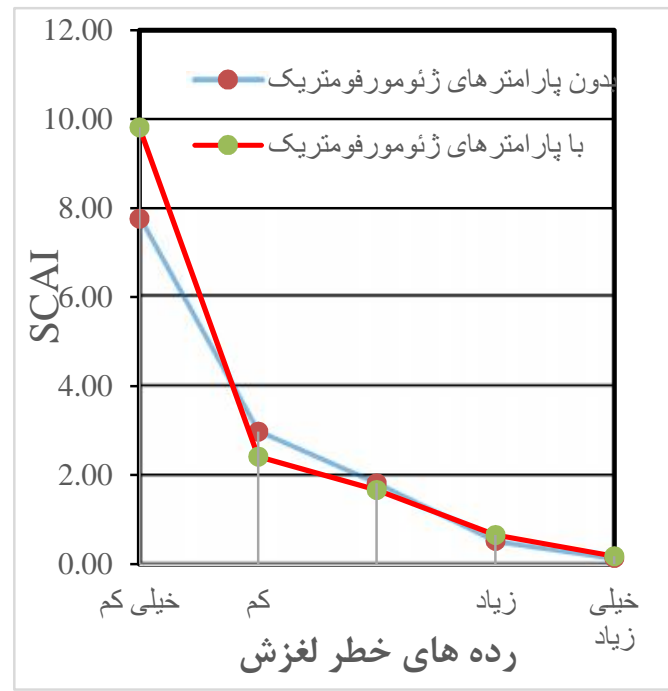

شكل ^- روند شاخص سطح سلول هسته Figure 8. Seed Cell Area Index Trend

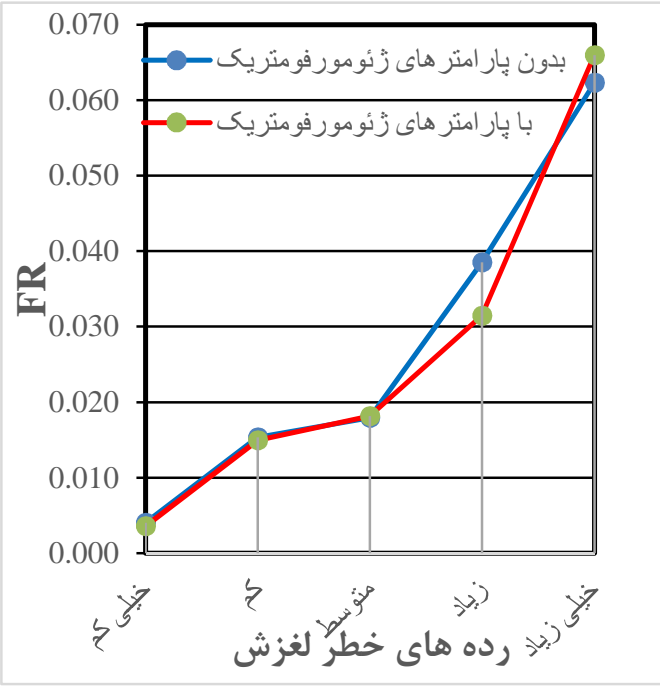

شكل V- روند شاخص نسبت فراوانى

Figure 7. Frequency Ratio Trend

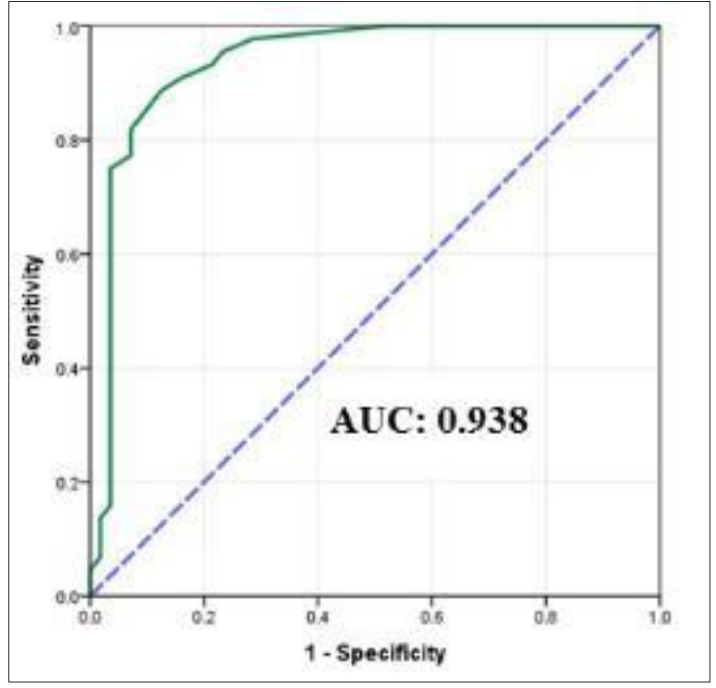

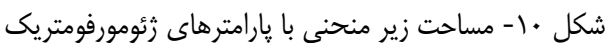
Figure 10. Area Under Curve with Geomorphometric Factors

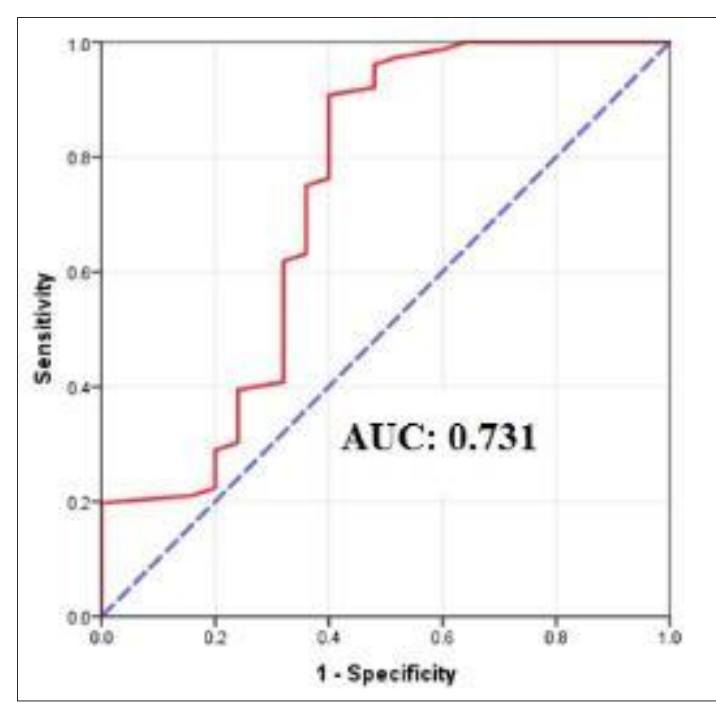

شكل 9- مساحت زير منحنى بدون يارامترهاى زئومورفومتريك . Figure 9. Area Under Curve without Geomorphometric Factors
گرفتهاند. طبق نتايج حاصل از يهناهبندى حساسيت خطر

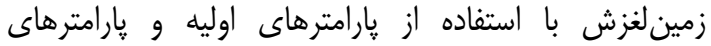

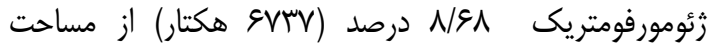

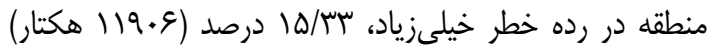

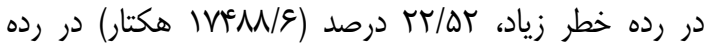
خطر متوسط، ه/ع

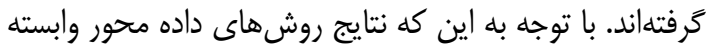

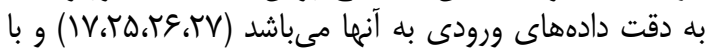

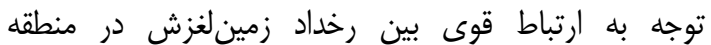

نتايج حاصل از يزوهش حاضر نشان داد كه يارامترهاى

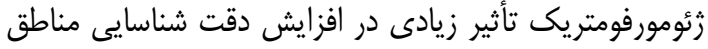

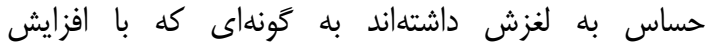

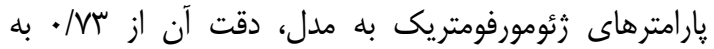

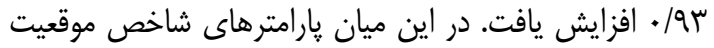

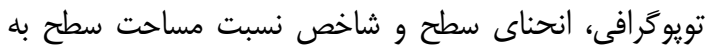

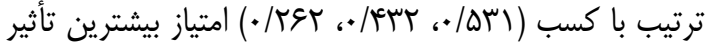

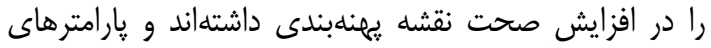

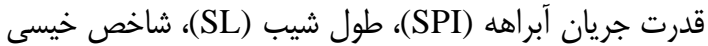
تويوگرافى (TWI) و انحناى مقطع در ردهان (SPI)، طول شعاى بعدى قرار 
$r$ r. ارزيابى كارايى بارامترهاى كمى زئوموروفتريك در افزايش صحت نقشههاى بهنهبندى حساسيت زمينلغزش .

تأثير شايان توجه يارامترهاى زئومورفومتريك در افزايش

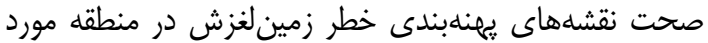

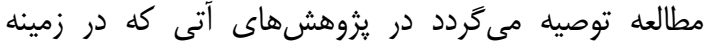

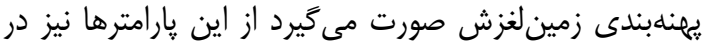

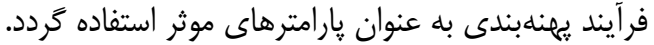

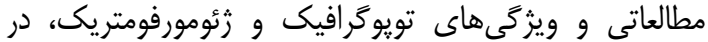

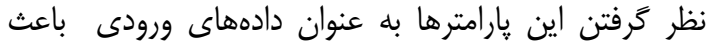

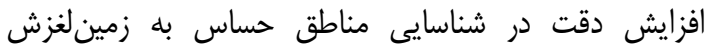

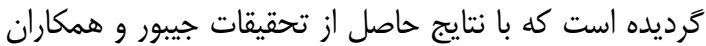

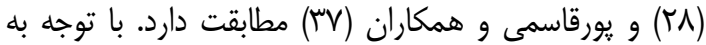

1. Arabameri, A.R. and A.H. Halabian. 2015. Landslide Hazard Zonation Using Statistical Model of AHP (Case Study: Zarand Saveh Basin), Physical Geomorphology, 28: 65-86.

2. Arabameri, A.R. and K. Shirani. 2016. Identification of Effective Factors on Landslide Occurrence and its Hazard Zonation Using Dempster-Shafer theory (Case study:Vanak Basin, Isfahan Province). Watershed Engineering and Management, 8(1): 93-106.

3. Arabameri, A.R. and K. Shirani. 2016. Prioritization of Effective Factors on Landslide Occurrence and its Susceptibility Zonation Using Statistical Methods, A Case Study: Vanak catchment. Geodynamics Research International Bulletin, 3(05): 22-38.

4. Arabameri, A.R., K. Shirani and Kh. Rezaei. 2017. A Comparative Assessment between Weights-ofEvidence and Frequency Ratio Models for Landslide Hazard Zonation in Vanak Basin, Watershed Management Research, 8(15): 147-160.

5. Arabameri, A.R., H.R. Pourghasemi, M. Yamani. 2017. Applying different scenarios for landslide spatial modeling using computational intelligence methods. Environmental Earth Sciences, 76: 832.

6. Althuwaynee, O.F., B. Pradhan and S. Lee. 2016. A novel integrated model for assessing landslide susceptibility mapping using CHAID and AHP pair-wise comparison. International Journal of Remote Sensing, 37: 1190-1209.

7. Armed Forces Geographical Organization, 1:50000 Topographic maps, Chqagrg 59561V, Sary Valley 5556II, Pare Pit 59551V, Hercan shit 5955I, Fereydoon shahr 50551V.

8. Atkinson, P.M. and R. Massari. 2011. Autologistic modelling of susceptibility to landsliding in the Central Apennines. Geomorphology, 130: 55-64.

9. Chalkias, C., M. Ferentinou and C. Polykretis. 2014. GIS-Based Landslide Susceptibility Mapping on the Peloponnese Peninsula. Geosciences, 4: 176-190

10. Chen, Z. and W. Jinfei. 2007. Landslide hazard mapping using logistic regression model in Machenzie Valley. Natural Hazard, 42: 75-89.

11. Chen, W., H. Chai, X. Sun, Q. Wang, X. Ding and H. Hong. 2016a. A GIS-based comparative study of frequency ratio, statistical index and weights-of-evidence models in landslide susceptibility mapping. Arabian Journal of Geosciences, 9: 1-16.

12. Chen, W., X. Ding, R. Zhao and S. Shi. 2016b. Application of frequency ratio and weights of evidence models in landslide susceptibility mapping for the Shangzhou District of Shangluo City, China. Environment Earth Sciences, 75:1-10.

13. Chen, W., W. Li, H. Chai, E. Hou, X. Li and X. Ding. 2016c. GIS-based landslide susceptibility mapping using analytical hierarchy process (AHP) and certainty factor (CF) models for the Baozhong region of Baoji City, China. Environment Earth Sciences, 75:1-14.

14. Chousianitis, K., V. Del Gaudio, N. Sabatakakis, K. Kavoura, G. Drakatos, G.D. Bathrellos and H.D. Skilodimou. 2016. Assessment of Earthquake-Induced Landslide Hazard in Greece: From Arias Intensity to Spatial Distribution of Slope Resistance Demand.Bulletin of the Seismological Society of America, 106:174-188.

15. Constantin, M., M. Bednarik, M.C. Jurchescu and M. Vlaicu. 2011. Landslide susceptibility assessment using the bivariate statistical analysis and the index of entropy in the Sibiciu Basin (Romania). Environment Earth Sciences, 63: 397-406.

16. Dikau, R. 1989. The application of a digital relief model to landform analysis in geomorphology. In: Raper, J. (Ed.), Three Dimensional Applications in Geographical Information Systems, Taylor and Francis, London: 51-77.

17. Dolatkhahi, Z., M.R. Javadi and M. Vafakhah. 2017. Using of two and multi variate regression models on landslide hazard zonation (a case study: North Tehran Watershed). Watershed Mmanagement Research, 8 (15): 171-179.

18. Dymond, J.R., A.G. Ausseil, J.D. Shepherd and L. Buettner. 2006. Validation of a region-wide model of landslide susceptibility in the Manawatu-Wanganui region of New Zealand. Geomorphology, 74: 70-79.

19. Evans, I.S. 1972. General Geomorphology, Derivatives of Altitude and Descriptive Statistics. In R.J. Chorley (Ed.), Spatial Analysis in Geomorphology: 17-90.

20. Geological Survey of Iran. 1998. 1:250000 Geological maps, Golpayegan and Shahrkord sheets.

21. Guzzetti, F., A.C. Mondini, M. Cardinali, F. Fiorucci, M. Santangelo and K.T. Chang. 2012. Landslide inventory maps: New tools for an old problem. Earth-Science Reviews, 112: 42-66.

22. Hengl, T., S. Gruber and D.P. Shrestha. 2003. Digital terrain analysis in ILWIS. International Institute for Geo-Information Science and Earth Observation Enschede. Netherlands: 62.

23. Holloway, J., C.A. Rudy, S.F. Lamoureux and P.M. Treitz. 2017. Determining the terrain characteristics related to the surface expression of subsurface water pressurization in permafrost landscapes using susceptibility modeling, The Cryosphere, 11: 1403-1415.

24. Hong, H., B. Pradhan, M.N. Jebur, D.T. Bui, C. Xu and A. Akgun. 2015. Spatial prediction of landslide hazard at the Luxi area (China) using support vector machines. Environmental Earth Sciences, 75(40). 
25. Hong, H., H.R. Pourghasemi and Z.S. Pourtaghi. 2016. Landslide susceptibility assessment in Lianhua County (China): a comparison between a random forest data mining technique and bivariate and multivariate statistical models. Geomorphology, 259: 105-118.

26. Hong, H., W. Chen, C. Xu, A.M. Youssef, B. Pradhan and D. Tien Bui. 2016. Rainfall-induced landslide susceptibility assessment at the Chongren area (China) using frequency ratio, certainty factor, and index of entropy. Geocarto International, 23(2): 139-154.

27. Hong, H., W. Chen, C. Xu, A.M. Youssef, B. Pradhan, and D. Tien Bui. 2017. Rainfall-induced landslide susceptibility assessment at the Chongren area (China) using frequency ratio, certainty factor, and index of entropy. Geocarto International, 32: 139-154.

28. Jebur, M.N., B. Pradhan and M.S. Tehrany. 2014. Optimization of landslide conditioning factors using very highresolution airborne laser scanning (LiDAR) data at catchment scale. Remote Sensing of Environmental, 152: 150-165.

29. Jenness, J. 2002. Surface Areas and Ratios from Elevation Grid. Jenness Enterprises.

30. Lee, S. and B. Pradhan. 2007. Landslide hazard mapping at Selangor, Malaysia using frequency ratio and logistic regression models. Landslides, 4: 33-41.

31. Moore, I.D., R.B. Grayson and A.R. Ladson. 1991. Digital terrain modeling: a review of hydrological. Geomorphological and biological applications, Hydrology Process, 5: 3-30.

32. National mapping agency. 1997. 1:40000 Aerial photos, Pishkuh Fereydūnshahr region.

33. Oh, H.J. and B. Pradhan. 2011. Application of a neuro-fuzzy model to landslide-susceptibility mapping for shallow landslides in a tropical hilly area. Computer Geosciences, 37(9:1264-1276).

34. Oh, H.J. and S. Lee. 2017. Shallow Landslide Susceptibility Modeling Using the Data Mining Models Artificial Neural Network and Boosted Tree, Applied science, 7: 1000.

35. Pawluszek, K. and A. Borkowski. 2017. Impact of DEM-derived factors and analytical hierarchy process on landslide susceptibility mapping in the region of Rozno'w Lake, Poland, Natural Hazards,
86: 919-952.

36. Pike, R.J., I.S. Evans and T. Hengl. 2009. Geomorphometry: A Brief Guide. Developments in Soil Science, 33(1)

37. Pourghasemi, H.R., H.R. Moradi, S.F. Aghda, C. Gokceoglu and B. Pradhan. 2014. GIS-based landslide susceptibility mapping with probabilistic likelihood ratio and spatial multi-criteria evaluation models (North of Tehran, Iran). Arabian Journal of Geosciences 7(5):1857-1878

38. Rasai, A., K. Khosravi, M. Habibnejad Roshan, A. Heidari and A. Mashayekh Khan. 2015. Lnadslide Hazard Zonation using Multivariate Regression in GIS Environment (Case Study: Aghmashhad Watershed, Mazandaran). Journal of watershed management research, 6 (12): 205-215.

39. Regmi, N.R., J. Giardio and J. Vitek. 2010. Modeling susceptibility to landslides using the weight of evidence: Western Colorado, USA. Geomorphology, 115: 172-187.

40. Shirani. K. 2003. Evaluation of the most important of zonation hazard landslide methods for selection appropriate method in south of Isfahan Province (Case Study: Semirom Region across Marbor River). Final report of research plan. Record No. 83/961.

41. Shirani, K and A.R. Arabameri. 2015. Landslide Hazard Zonation Using Logistic Regression Method (Case Study: Dez-e-Oulia Basin), journal of water and soil science, 72: 321-334.

42. Shirani, K., F. Heydari, A.R. Arabameri. 2017. Landslide Risk Zoning Potential by Analytical Comparison of artificial neural network and multivariate regression methods in landslide hazard zonation, case study: Vanak Basin. Watershed Engineering and Management, 9(4): 45-464.

43. Tagil, S. and J. Jenness. 2008. GIS-based automated landform classification and topographic, land cover and geologic attributes of landforms around the Yazoren Poje. Turkey. Applied Sciences, 8(6): 910-921.

44. Talebi, A., R. Uijlenhoet and P.A. Troch. 2007. Soil moisture storage and hillslope stability. Nat Hazards Earth System Science, 7: 523-534.

45. Taze, M., M. Asadi and S. Kalantari. 2015. Evaluation of capacity the Geomorphomety indicators in extracting drainage map (Seghale Sambusarayan water catchment). Quantitative Geomorphological Research, 1:134-144

46. Taze, M., S. Kalantari, H. Fathizad, R. Taghizade Mehrherdi. 2014. Classification of glacis based on Geomorphomety parameters (Aghda, Yazd). Quantitative Geomorphological Research, 4: 105-116.

47. Tobler, W.R. 1976. Analytical cartography, The American Cartographer, 3(1): 21-31.

48. Van Western, C.J., T.H. Van Asch and R. Soeters, 2005. Landslide Hazard and Risk Zonation: Why is it Still so Difficult, Bulletin of Engineering Geology and the Environment, 2: 176-184.

49. Wang, Q., D. Wang, Y. Huang, Z. Wang, L. Zhang, Q. Guo, W. Chen, W. Chen and M. Sang. 2015. Landslide Susceptibility Mapping Based on Selected Optimal Combination of Landslide Predisposing Factors in a Large Catchment. Sustainability7: 16653-16669.

50. Yesilnacar, E.K. 2005. The application of computational intelligence to landslide susceptibility mapping in Turkey, Ph.D Thesis. Department of Geomatics the University of Melbourne: 423 pp.

51. Yilmaz, I. 2009. Landslide susceptibility mapping using frequency ratio, logistic regression, artificial neural networks and their comparison: A case study from Kat landslides (Tokat-Turkey). Computers and Geosciences, 35: 1125-1138.

52. Zhou, S., G. Chen, L. Fang and Y. Nie. 2016. GIS-Based Integration of Subjective and Objective Weighting Methods for Regional Landslides Susceptibility Mapping. Sustainability, 8(334).

53. Zhou, S. and L. Fang. 2015. Support vector machine modeling of earthquake-induced landslides susceptibility in central part of Sichuan province. Geoenvironmental disasters, 2(2). 


\title{
Evaluating of Quantitative Geomorphometric Parameters Efficiency in Increasing the Accuracy of Landslide Sensitivity Maps (Case Study: Fereydoun Shahr Basin, Isfahan Province)
}

\author{
Alireza Arabameri ${ }^{1}$, Khalil Rezaei ${ }^{2}$, Masoud Sohrabi ${ }^{3}$ and Kourosh Shirani ${ }^{4}$ \\ 1- PhD of Geomorphology, Faculty of Geomorphology, Tarbiat Modarres University \\ (Corresponding Author: Alireza.ameri91@yahoo.com) \\ 2- Assistant Professor of sedimentology, Geology Department, Kharazmi University, Tehran. \\ 3- M.Sc. Department Of Civil Engineering,Urmia Branch,Islamic Azad University,Urmia,Iran. \\ 4- Assistant Professor, Soil Conservation and Watershed Management Research Department, Isfahan Agricultural \\ and Natural Resources, Research and Education Center, AREEO, Isfahan, Iran. \\ Received: 13 July, 2017 \\ Accepted: 21 Apr, 2018
}

\begin{abstract}
One of the goals of geomorphologists in working with the models of different landforms is to obtain better relations in realizing the physical realities of environment. In this study, to evaluate the performance of geomorphometric parameters to increase accuracy of zoning landslide susceptibility map has been studied. As the first step by the application of nine initial conditioning factors including slope, aspect, elevation, land use, lithology, distance from roads, rivers and vegetation index (NDVI) the zoning map was provided. In the next step geomorphometric parameters influential on the occurrence of landslide including topographic location index (TPI), surface curvature, curved sections, slope length (LS), Topographic wetness index (TWI), stream flow power (SPI), surface area ration index (SAR), was added to the model and then the zoning map was obtained. In the final step, the zoning maps was evaluated by using ROC curve. To provide zoning maps a new mixed model was applied, so, for determination of criteria weights multivariate regression and to determine weight of the classes' frequency ratio method was utilized. The findings of this research indicated that geomorphometric factors have a considerable influence on the increase of identification of regions that are susceptible to the landslides and enhance the accuracy of zoning maps from 0.731 to 0.938 . These factors have also increased the resolution of the slip classes. According to the results, topography position index, plan curvature and surface area ratio have the highest influence on the accuracy of zoning maps. Based on superior approach, 8.68\% (6737 ha) of the region are at very high risk and $15.3 \%$ (11906 ha) have been identified as high risk areas. According to the high ability of geomorphologic parameters in the identification of susceptible areas to the landslide, the application of these parameters is recommended in landslide hazard zonation.
\end{abstract}

Keywords: Geomorphometry, Validation, Zonation, Landslide, Isfahan Province 